\title{
Duality in infinite dimensional linear programming
}

\section{H. Edwin Romeijn}

Department of Operations Research \& Tïbergen Institute, Erasmus University Rotterdam, 3000 DR Rotterdam, Netherlands

\section{Robert L. Smith and James C. Bean}

Department of Industrial and Operations Engineering, The University of Michigan, Ann Arbor, MI 48109-2117, USA

Received 20 December 1989

Revised manuscript received 17 May 1990

We consider the class of linear programs with infinitely many variables and constraints having the property that every constraint contains at most finitely many variables while every variable appears in at most finitely many constraints. Examples include production planning and equipment replacement over an infinite horizon. We form the natural dual linear programming problem and prove strong duality under a transversality condition that dual prices are asymptotically zero. That is, we show, under this transversality condition, that optimal solutions are attained in both primal and dual problems and their optimal values are equal. The transversality condition, and hence strong duality, is established for an infinite horizon production planning problem.

Key words: Infinite dimensional linear program, duality, infinite horizon optimization.

\section{Introduction}

Consider the following doubly infinite linear programming problem:

$$
\min \sum_{i=1}^{\infty} c_{i}^{\prime} x_{i}
$$

subject to

$$
\begin{aligned}
& A_{11} x_{1} \geqslant b_{1}, \\
& A_{i, i-1} x_{i-1}+A_{i i} x_{i} \geqslant b_{i} \quad(i=2,3, \ldots), \\
& 0 \leqslant x_{i} \leqslant u_{i} \quad(i=1,2, \ldots),
\end{aligned}
$$

This material is based on work supported by the National Science Foundation under Grant No. ECS-8700836. 
and its natural dual

$$
\max \sum_{i=1}^{\infty}\left(b_{i}^{\prime} y_{i 1}-u_{i}^{\prime} y_{i 2}\right)
$$

subject to

$$
\begin{aligned}
& A_{i i}^{\prime} y_{i 1}+A_{i+1, i}^{\prime} y_{i+1,1}-y_{i 2} \leqslant c_{i} \quad(i=1,2, \ldots), \\
& y_{i j} \geqslant 0 \quad(i=1,2, \ldots ; j=1,2),
\end{aligned}
$$

where $c_{i}, u_{i}, x_{i}, y_{i 2} \in \mathbb{R}^{n_{i}}$ and $b_{i}, y_{i 1} \in \mathbb{R}^{m_{i}}$ are all column vectors, and $A_{i j}$ is an $\left(m_{i} \times n_{j}\right)$-matrix. The problem (P) represents the class of all (bounded) linear programs having the property that every constraint contains at most finitely many variables and (in a dual fashion) every variable appears in at most finitely many constraints (Schochetman and Smith, 1992). Applications include infinite horizon production planning under nonstationary demand, equipment replacement under technological change, and capacity expansion. In this paper, we establish a theory of duality for $(\mathrm{P})$ and $(\mathrm{D})$ by providing conditions under which both weak and strong duality hold.

Historically, abstract duality theory allowing for consideration of the infinite dimensional case began with the fundamental paper of Duffin (1956). Charnes, Cooper and Kortanek (1963) subsequently stated and proved a strong duality theorem for semi-infinite linear programming using an extension of Farkas' lemma as proven by Haar. However, as later pointed out by Duffin and Karlovitz (1965), they failed in that paper to explicitly include an interior point condition in whose absence a duality gap may exist. The failure of strong and even weak duality to hold in the infinite dimensional case in the absence of interior point conditions (Luenberger, 1969, p. 217) or, more generally, closedness (Anderson and Nash, 1987, p. 52; Ponstein, 1980), has kept much of the literature at an abstract level. The difficulty has been in establishing these conditions in concrete cases. Much of the success thus far has in fact been limited to the semi-infinite case, introduced in Charnes, Cooper and Kortanek (1963), and subsequently developed in Ben-Israel, Charnes and Kortanek (1969), Karney (1981), Borwein (1981, 1983), and Duffin, Jeroslow and Karlovitz (1983). The so-called separably infinite programs introduced in Charnes, Gribek and Kortanek (1980) allow an infinite number of variables and constraints, although no infinite subset of constraints is allowed to contain more than finitely many distinct variables. Programs that are truly doubly infinite are excluded and in particular, (P) is not included in this class.

Work on the doubly infinite case includes Evers (1973, 1983), Hopkins (1971), Grinold (1971, 1977), and Jones, Zydiak and Hopp (1988). Grinold (1971) provides sufficient conditions for the existence of optimal primal and dual solutions for a special class of doubly infinite problems and establishes a weak duality theorem for a stationary infinite stage LP in Grinold (1977). This latter work was extended to convex programs in Grinold (1983). Jones, Zydiak and Hopp (1988) applies the 
general theory developed in Grinold and Hopkins (1972) to a cost stationary infinite horizon equipment replacement problem with time varying demand to establish the existence of optimal stationary dual solutions.

Our approach as in Grinold $(1971,1977,1983)$ is to establish properties for (P) and (D) indirectly through the inheritance of such properties from finite dimensional approximations of (P) and (D). These are formed by truncating beyond finitely many variables and constraints. This approach avoids the necessity of establishing closedness or interior point properties for (P) or (D) directly. Viewing the index $i$ in $(\mathrm{P})$ as corresponding to the $i$ th period in a multiperiod planning problem, the above truncation to $(\mathrm{P})$ becomes a finite horizon approximation to an infinite horizon problem. This so-called planning or solution horizon approach to the analysis of (P) has an extensive literature (for more recent work, see, e.g., Bean and Smith, 1984; Bès and Sethi, 1988; and Schochetman and Smith, 1989).

Throughout the paper, we adopt the following assumptions.

Assumption A. The set, $X$, of feasible solutions to (P) is nonempty, i.e., $X \neq \emptyset$.

Assumption B. The objective function $C(x)=\sum_{i=1}^{\infty} c_{i}^{\prime} x_{i}$ in $(\mathrm{P})$ is uniformly convergent over $x$, i.e., $\sum_{i=1}^{\infty}\left\|c_{i}\right\|_{\infty}<\infty$ where $\left\|c_{i}\right\|_{\infty}=\max \left\{\left|c_{i}^{\prime} x\right|: 0 \leqslant x \leqslant u_{i}\right\}$.

A sufficient condition for Assumption B to hold is that each $c_{i}$ be of the form $\alpha^{i} k_{i}$ where $0<\alpha<1$, and the $k_{i}$ and $u_{i}$ are uniformly bounded ( $\alpha$ corresponds here to a discount factor).

In Section 2, we establish topological spaces within which to embed (P) and (D) and thereby establish that $(\mathrm{P})$ has an optimal solution. We also formally define the finite dimensional truncations $(\mathrm{P}(N))$ and $(\mathrm{D}(N))$ consisting of the first $N$ variables and $N$ constraints of (P) and (D) respectively.

In Section 3, weak duality is established for the pair (P) and (D) under the condition that the off diagonal submatrices $A_{i+1, i}$ are eventually nonnegative for all i. Moreover, whenever weak duality holds, we show that no duality gap exists, i.e., the infimal value of the primal program $(P)$ equals the supremal value of the dual program (D).

In Section 4, strong duality is established under a transversality condition requiring that the optimal dual multiplier associated with the $i$ th constraint converges to zero as $i$ goes to infinity. Roughly speaking, we require that the optimal prices of future resources become arbitrarily small. Under this condition, an optimal dual solution is shown to exist at which primal and dual objective values are equal and complementary slackness holds.

Finally, in Section 5, as an illustration of the general theory, we establish strong duality under mild regularity conditions for a general nonstationary infinite horizon production planning problem. 


\section{Mathematical preliminaries}

We begin by forming the product spaces $\prod_{i=1}^{\infty} \mathbb{R}^{n_{i}}$ and $\prod_{i=1}^{\infty} \mathbb{R}^{m_{i}+n_{i}}$ within which we embed (P) and (D) respectively. Each is equipped with the corresponding product topologies inherited from the underlying Euclidean spaces so that for example a sequence $\left\{x^{n}\right\} \subseteq \prod_{i=1}^{\infty} \mathbb{R}^{n_{i}}$ converges precisely when its components $x_{i}^{n}$ converge in the Euclidean metric for all $i$. That is,

$$
x^{n} \rightarrow x \text { as } n \rightarrow \infty \text { if and only if } x_{i}^{n} \rightarrow x_{i} \text { as } n \rightarrow \infty,
$$

for all $i=1,2, \ldots$. Similarly for $\left\{y^{n}\right\} \subseteq \prod_{i=1}^{\infty} \mathbb{R}^{m_{i}+n_{i}}$. Note that the nonnegative orthant has an empty interior in both spaces, so that interior point conditions do not hold here.

Since the feasible region for $(\mathrm{P}), X$, is closed and contained within the compact set $\prod_{i=1}^{\infty}\left[0, u_{i}\right]$, it is also compact and, by Assumption A, nonempty. By Assumption $\mathrm{B}$, the objective function $C(x)=\sum_{i=1}^{\infty} c_{i}^{\prime} x_{i}$ is a continuous function over $X$ (Schochetman and Smith, 1989). It follows that an optimal solution $x^{*}$ to (P) exists.

Since the objective function in (D) may fail to converge for some feasible $y \in \prod_{i=1}^{\infty} \mathbb{R}^{m_{i}+n_{i}}$, we shall for now replace the objective function in (D) by

$$
B(y)=\limsup _{N \rightarrow \infty} \sum_{i=1}^{N}\left(b_{i}^{\prime} y_{i 1}-u_{i}^{\prime} y_{i 2}\right) \text {. }
$$

We shall see later that the two objective functions are in agreement over a subset of the feasible solutions $Y$ to (D) known to contain any optimum.

We shall establish duality results for $(\mathrm{P})$ and $(\mathrm{D})$ by demonstrating their inheritance from finite dimensional approximations $(\mathrm{P}(N))$ and $(\mathrm{D}(N))$. These are formed by dropping all variables and constraints beyond the first $N$ of (P) and (D), respectively. More formally, we define $(\mathrm{P}(N))$ by

$$
(\mathrm{P}(N)) \min \sum_{i=1}^{N} c_{i}^{\prime} x_{i}
$$

subject to

$$
\begin{aligned}
& A_{11} x_{1} \geqslant b_{1}, \\
& A_{i, i-1} x_{i-1}+A_{i i} x_{i} \geqslant b_{i} \quad(i=2,3, \ldots, N), \\
& 0 \leqslant x_{i} \leqslant u_{i} \quad(i=1,2, \ldots, N),
\end{aligned}
$$

and $(\mathrm{D}(N))$ by

$$
(\mathrm{D}(N)) \max \sum_{i=1}^{N}\left(b_{i}^{\prime} y_{i 1}-u_{i}^{\prime} y_{i 2}\right)
$$

subject to

$$
\begin{aligned}
& A_{i i}^{\prime} y_{i 1}+A_{i+1, i}^{\prime} y_{i+1,1}-y_{i 2} \leqslant c_{i} \quad(i=1,2, \ldots, N-1), \\
& A_{N N}^{\prime} y_{N 1}-y_{N 2} \leqslant c_{N}, \\
& y_{i j} \geqslant 0 \quad(i=1,2, \ldots N ; j=1,2) .
\end{aligned}
$$


Note that $(\mathrm{D}(N))$ is the ordinary linear programming dual of $(\mathrm{P}(N))$ so that classical weak and strong duality holds for each pair $(\mathrm{P}(N)),(\mathrm{D}(N))$ for all $N$. That is, by weak duality, we have that $B(y ; N) \leqslant C(x ; N)$ for all $x \in X(N), y \in Y(N)$ and for all $N$, where $B(y ; N)$ and $C(x ; N)$ and $Y(N)$ and $X(N)$ are the objective functions and feasible regions of $(\mathrm{D}(N))$ and $(\mathrm{P}(N))$ respectively. Here $X(N)$ is regarded as a subset of $\prod_{i=1}^{\infty} \mathbb{R}^{n_{i}}$ with the first $N$ elements arbitrarily extended to elements of $\prod_{i=1}^{\infty} \mathbb{R}^{n_{i}}$. It will also, at times, be convenient to think of $X(N)$ as a subset of $\prod_{i=1}^{N} \mathbb{R}^{n_{i}}$. We shall use the same notation for both where the interpretation should be clear from the context. Similarly, for $Y(N)$. Strong duality provides that $B^{*}(N)=C^{*}(N)$ for all $N$ where $B^{*}(N)$ and $C^{*}(N)$ are the optimal values to linear programs $(\mathrm{D}(N))$ and $(\mathrm{P}(N))$ respectively. Note that $(\mathrm{P}(N))$ has an optimal solution for all $N$ since its feasible region is nonempty and compact (since $X \subseteq X(N)$ for all $N$ ) and its objective function is continuous.

In the next section, we construct a counterexample to weak duality for the pair (P) and (D) and provide a sufficient condition for weak duality to hold. We end this section with a summary of notation.

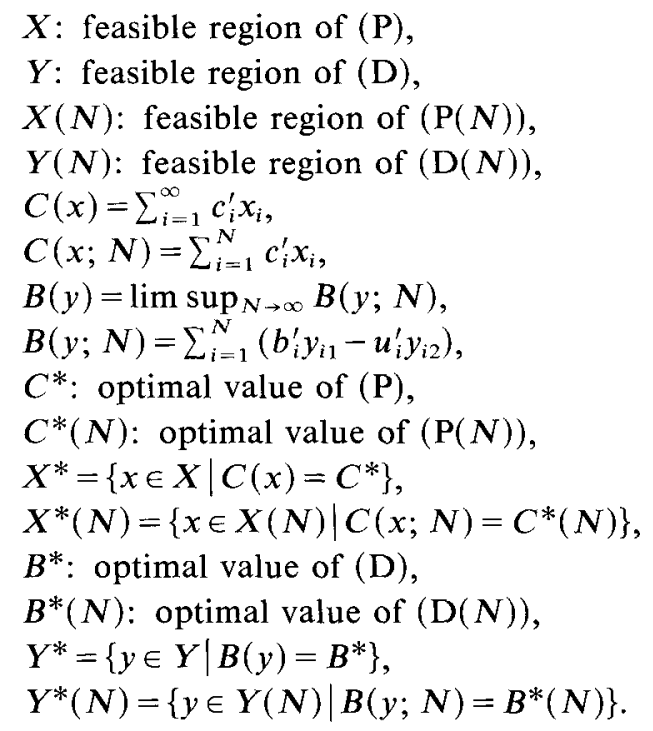

\section{Weak duality}

Because the feasible region to (D) is unbounded, weak duality for the pair (P) and (D) may fail to hold. Consider for example the following instance of $(P)$ :

$$
\min \sum_{i=1}^{\infty}\left(\frac{1}{2}\right)^{i-1} z^{i}
$$


subject to

$$
\begin{aligned}
& x_{1} \geqslant 1, \\
& y_{1}+z_{1} \geqslant 1, \\
& -2 y_{i-1}+x_{i} \geqslant 0 \quad(i=2,3, \ldots), \\
& -2 x_{i-1}+y_{i}+z_{i} \geqslant 0 \quad(i=2,3, \ldots), \\
& 0 \leqslant x_{i} \leqslant 2^{i-1} \quad(i=1,2, \ldots), \\
& 0 \leqslant y_{i} \leqslant 2^{i-1} \quad(i=1,2, \ldots), \\
& 0 \leqslant z_{i} \leqslant 1 \quad(i=1,2, \ldots),
\end{aligned}
$$

so that (D) is given by

(D)

$$
\sup \limsup _{N \rightarrow \infty}\left(u_{1}+v_{1}-\sum_{i=2}^{N}\left(2^{i} p_{i}+2^{i} q_{i}+r_{i}\right)\right)
$$

subject to

$$
\begin{aligned}
& u_{i}-2 v_{i+1}-p_{i} \leqslant 0 \quad(i=1,2, \ldots), \\
& v_{i}-2 u_{i+1}-q_{i} \leqslant 0 \quad(i=1,2, \ldots), \\
& v_{i}-r_{i} \leqslant\left(\frac{1}{2}\right)^{i-1} \quad(i=1,2, \ldots), \\
& u_{i}, v_{i}, p_{i}, r_{i} \geqslant 0 \quad(i=1,2, \ldots) .
\end{aligned}
$$

It is a simple matter to verify that $(\mathrm{P})$ satisfies Assumptions A and B. However, the following solution is optimal for $(P)$ with value 0 :

$$
\begin{aligned}
& x_{i}=2^{i-1} \quad(i=1,2, \ldots), \\
& y_{i}=2^{i-1} \quad(i=1,2, \ldots), \\
& z_{i}=0 \quad(i=1,2, \ldots),
\end{aligned}
$$

while the following solution is optimal for (D) with value 2 :

$$
\begin{aligned}
& u_{i}=\left(\frac{1}{2}\right)^{i-1} \quad(i=1,2, \ldots), \\
& v_{i}=\left(\frac{1}{2}\right)^{i-1} \quad(i=1,2, \ldots), \\
& p_{i}=q_{i}=r_{i}=0 \quad(i=1,2, \ldots) .
\end{aligned}
$$

Hence weak duality fails for this instance of $(P)$. It is interesting that weak duality holds and is easily shown when (D) is defined as the algebraic dual of (P) (Anderson and Nash (1987, p. 18)). However a concrete representation of algebraic duals is usually unattainable in the infinite dimensional case. Evidently, (D) as given here is not such a representation. In fact, its objective function is not a linear functional.

The pathology exhibited above can be eliminated by requiring that all feasible solutions of (D) be feasible for $(\mathrm{D}(N))$, i.e.,

$$
Y \subseteq Y(N)
$$

for large $N$. The following theorem provides a sufficient condition for this to occur. 
Theorem 3.1. Suppose that $A_{i+1, i}$ is eventually nonnegative, i.e. there exists a positive integer $N^{\prime}$ such that $A_{i+1, i} \geqslant 0$ for all $i \geqslant N^{\prime}$. Then

$$
B(y) \leqslant C(x)
$$

for all $x \in X, y \in Y$.

Proof. If $y \in Y$, then we have for all $N \geqslant N^{\prime}$,

$$
c_{N} \geqslant A_{N N}^{\prime} y_{N 1}+A_{N+1, N}^{\prime} y_{N+1,1}-y_{N 2} \geqslant A_{N N}^{\prime} y_{N 1}-y_{N 2}
$$

since $y_{N+1,1} \geqslant 0$ and $\boldsymbol{A}_{N+1, N}$ contains only nonnegative elements. Hence $y$ is also feasible for $(\mathrm{D}(N))$, i.e., $y \in Y(N)$, for all $N \geqslant N^{\prime}$. By weak duality for finite dimensional LP-problems we have

$$
B(y ; N) \leqslant \min _{x \in X(N)} C(x ; N)=C^{*}(N)
$$

for all $y \in Y, N \geqslant N^{\prime}$. Our assumptions on the function $C(\cdot)$ imply that value convergence holds for (P), i.e., $\lim _{N \rightarrow \infty} C^{*}(N)$ exists and is equal to $C^{*}$ (Schochetman and Smith, 1992). Therefore we have

$$
B(y)=\limsup _{N \rightarrow \infty} B(y ; N) \leqslant \lim _{N \rightarrow \infty} C^{*}(N)=C^{*} \leqslant C(x)
$$

for all $x \in X, y \in Y$.

Fortunately, the nonnegativity condition on the off diagonal elements $A_{i+1, i}$ is not restrictive in most multistage planning problems where $A_{i+1, i}$ corresponds to inventory carryover from the previous period.

Theorem 3.1 tells us that every feasible value of the primal (P) will be an upper bound to every feasible value or the dual (D). The next result shows that the supremum of the latter equals the infimum (i.e., minimum) of the former. We summarize this claim by saying that no duality gap exists.

Theorem 3.2 (No Duality Gap). Suppose weak duality holds, i.e., $B(y) \leqslant C(x)$ for all $x \in X, y \in Y$. Then

$$
B^{*}=\sup _{y \in Y} B(y)=\min _{x \in X} C(x)=C^{*} .
$$

Moreover, value convergence holds for (D), i.e., $B^{*}=\lim _{N \rightarrow \infty} B^{*}(N)$.

Proof. Consider $y^{*}(N) \in Y^{*}(N)\left(y^{*}(N)\right.$ is an optimal solution of $\left.(D(N))\right)$. Then define $z^{N}$ as

$$
\begin{aligned}
& z_{i j}^{N}=y_{i j}^{*}(N) \quad(i=1, \ldots, N ; j=1,2), \\
& z_{i 1}^{N}=0 \quad(i=N+1, \ldots), \\
& z_{i 2}^{N}=\max \left(\theta,-c_{i}\right) \quad(i=N+1, \ldots),
\end{aligned}
$$


where $f=\max (d, e)\left(d, e, f \in \mathbb{R}^{n}\right)$ is defined as follows:

$$
f_{i}=\max \left(d_{i}, e_{i}\right) \quad(i=1,2, \ldots, n),
$$

and where $\theta=(0, \ldots, 0)^{\prime}$. Obviously, $z^{N}$ satisfies the first $N-1$ constraints of (D). Furthermore,

$$
A_{N N}^{\prime} z_{N 1}^{N}+A_{N+1, N}^{\prime} z_{N+1,1}^{N}-z_{N 2}^{N}=A_{N N}^{\prime} y_{N 1}^{*}(N)-y_{N 2}^{*}(N) \leqslant c_{N}
$$

and

$$
A_{i i}^{\prime} z_{i 1}^{N}+A_{i+1, i}^{\prime} z_{i+1,1}^{N}-z_{i 2}^{N}=-\max \left(\theta,-c_{i}\right)=\min \left(\theta, c_{i}\right) \leqslant c_{i}
$$

for $i=N+1, \ldots$ Thus, $z^{N} \in Y$. Note also that $z^{N} \in Y^{*}(N)$. Now we have

$$
\begin{aligned}
B^{*} & =\sup _{y \in Y} B(y) \\
& \geqslant B\left(z^{N}\right) \\
& =\limsup _{M \rightarrow \infty}\left(\sum_{i=1}^{M}\left(b_{i}^{\prime} z_{i 1}^{N}-u_{i}^{\prime} z_{i 2}^{N}\right)\right) \\
& =\limsup _{M \rightarrow \infty}\left(\sum_{i=1}^{N}\left(b_{i}^{\prime} z_{i 1}^{N}-u_{i}^{\prime} z_{i 2}^{N}\right)+\sum_{i=N+1}^{M}\left(b_{i}^{\prime} z_{i 1}^{N}-u_{i}^{\prime} z_{i 2}^{N}\right)\right) \\
& =\sum_{i=1}^{N}\left(b_{i}^{\prime} y_{i 1}^{*}(N)-u_{i}^{\prime} y_{i 2}^{*}(N)\right)+\limsup _{M \rightarrow \infty}\left(-\sum_{i=N+1}^{M} u_{i}^{\prime} \max \left(\theta,-c_{i}\right)\right) \\
& =B\left(y^{*}(N) ; N\right)-\liminf _{M \rightarrow \infty}\left(\sum_{i=N+1}^{M} u_{i}^{\prime} \max \left(\theta,-c_{i}\right)\right) \\
& =B^{*}(N)-\liminf _{M \rightarrow \infty}\left(\sum_{i=N+1}^{M} \sum_{j: c_{i j}<0}\left(-u_{i j} c_{i j}\right)\right) \\
& =C^{*}(N)-\sum_{i=N+1}^{\infty} \sum_{j: c_{i j}<0}\left(-u_{i j} c_{i j}\right)
\end{aligned}
$$

for all $N$. In the last step we have used the absence of a duality gap for finite dimensional LP, and the fact that $-u_{i j} c_{i j}>0$ for all $(i, j)$ such that $c_{i j}<0$. Since

$$
\begin{aligned}
\sum_{i=1}^{\infty} \sum_{j: c_{i j}<0}\left(-u_{i j} c_{i j}\right) & \leqslant \max \left(\sum_{i=1}^{\infty} \sum_{j: c_{i j}>0} u_{i j} c_{i j}, \sum_{i=1}^{\infty} \sum_{j: c_{i j}<0}\left(-u_{i j} c_{i j}\right)\right) \\
& \leqslant \sum_{i=1}^{\infty} \max \left(\sum_{j: c_{i j}>0} u_{i j} c_{i j}, \sum_{j: c_{i j}<0}\left(-u_{i j} c_{i j}\right)\right) \\
& =\sum_{i=1}^{\infty}\left\|c_{i}\right\|_{\infty}<\infty,
\end{aligned}
$$

we can conclude that

$$
\lim _{N \rightarrow \infty} \sum_{i=N+1}^{\infty} \sum_{j: c_{i j}<0}\left(-u_{i j} c_{i j}\right)=0 .
$$


Furthermore, $\lim _{N \rightarrow \infty} C^{*}(N)$ exists and is equal to $C^{*}$. So now we have

$$
B^{*} \geqslant C^{*} \text {. }
$$

By weak duality we have $B^{*}=\sup _{y \in X} B(y) \leqslant \inf _{x \in X} C(x)=C^{*}$, so we can conclude that

$$
B^{*}=C^{*}
$$

Also,

$$
\lim _{N \rightarrow \infty} B^{*}(N)=\lim _{N \rightarrow \infty} C^{*}(N)=C^{*}=B^{*}
$$

\section{Strong duality}

In this section, we establish conditions under which an optimal solution, $y^{*}$, exists to the dual program (D). Under weak duality and Theorem 3.2, it will follow that optimal primal and dual values are attained and equal, i.e., strong duality. The method will be to construct a candidate solution for $y^{*}$ from the set $Y^{*}(\infty)$ of accumulation points of finite dimensional dual optima $Y^{*}(N)$.

We begin by establishing that every pair of accumulation points $x^{*}$ and $y^{*}$ of corresponding finite dimensional optimal solutions are primal and dual feasible and moreover necessarily satisfy complementary slackness.

Lemma 4.1. Suppose $x^{*}(N) \in X^{*}(N), y^{*}(N) \in Y^{*}(N)$ are optimal solutions for $(\mathrm{P}(N))$ and $(\mathrm{D}(N))$, respectively, $N=1,2,3, \ldots$ Furthermore, assume that for some subsequence $\left\{N_{k}\right\}$ of the positive integers that is independent of $i$, we have

$$
\begin{aligned}
& \lim _{k \rightarrow \infty} x_{i}^{*}\left(N_{k}\right)=x_{i}^{*} \quad(i=1,2, \ldots), \\
& \lim _{k \rightarrow \infty} y_{i}^{*}\left(N_{k}\right)=y_{i}^{*} \quad(i=1,2, \ldots),
\end{aligned}
$$

i.e., $x^{*} \in X^{*}(\infty)$ and $y^{*} \in Y^{*}(\infty)$, the sets of accumulation points of $X^{*}(N)$ and $Y^{*}(N)$ respectively. Then $x^{*} \in X$ and $y^{*} \in Y$, i.e.,

$$
X^{*}(\infty) \subseteq X \text { and } Y^{*}(\infty) \subseteq Y .
$$

Furthermore, $x^{*}$ and $y^{*}$ satisfy complementary slackness, i.e.,

$$
\begin{aligned}
& \left(A_{i, i-1} x_{i-1}^{*}+A_{i i} x_{i}^{*}-b_{i}\right)^{\prime} y_{i 1}^{*}=0, \\
& \left(u_{i}-x_{i}^{*}\right)^{\prime} y_{i 2}^{*}=0
\end{aligned}
$$

and

$$
\left(c_{i}-A_{i i}^{\prime} y_{i 1}^{*}-A_{i+1, i}^{\prime} y_{i+1,1}^{*}+y_{i 2}^{*}\right)^{\prime} x_{i}^{*}=0
$$

for $i=1,2, \ldots$ 
Proof. The fact that $x^{*} \in X$ and $y^{*} \in Y$ follows immediately from the fact that every constraint is a linear function of finitely many variables. By complementary slackness for finite dimensional linear programming we have, for all $k$ :

$$
\begin{aligned}
& \left(A_{i, i-1} x_{i-1}^{*}\left(N_{k}\right)+A_{i i} x_{i}^{*}\left(N_{k}\right)-b_{i}\right)^{\prime} y_{i 1}^{*}\left(N_{k}\right)=0 \quad\left(i=1,2, \ldots, N_{k}\right), \\
& \left(u_{i}-x_{i}^{*}\left(N_{k}\right)\right)^{\prime} y_{i 2}^{*}\left(N_{k}\right)=0 \quad\left(i=1,2, \ldots, N_{k}\right)
\end{aligned}
$$

and

$$
\begin{aligned}
\left(c_{i}-A_{i i}^{\prime} y_{i 1}^{*}\left(N_{k}\right)-A_{i+1, i}^{\prime} y_{i+1,1}^{*}\left(N_{k}\right)+y_{i 2}^{*}\left(N_{k}\right)\right)^{\prime} x_{i}^{*}\left(N_{k}\right)=0 & \\
& \left(i=1,2, \ldots, N_{k}-1\right), \\
\left(c_{N_{k}}-A_{N_{k} N_{k}}^{\prime} y_{N_{k} 1}^{*}\left(N_{k}\right)+y_{N_{k} 2}^{*}\left(N_{k}\right)\right)^{\prime} x_{N_{k}}^{*}\left(N_{k}\right)= & 0 .
\end{aligned}
$$

Taking the limit for $k \rightarrow \infty$ in, for example, equation (2) yields for all $i$,

$$
\lim _{k \rightarrow \infty}\left(u_{i}-x_{i}^{*}\left(N_{k}\right)\right)^{\prime} y_{i 2}^{*}\left(N_{k}\right)=0
$$

or

$$
\left(u_{i}-\lim _{k \rightarrow \infty} x_{i}^{*}\left(N_{k}\right)\right)^{\prime}\left(\lim _{k \rightarrow \infty} y_{i 2}^{*}\left(N_{k}\right)\right)=0,
$$

thus

$$
\left(u_{i}-x_{i}^{*}\right)^{\prime} y_{i 2}^{*}=0 .
$$

Analogously we get for all $i$,

$$
\left(A_{i, i-1} x_{i-1}^{*}+A_{i i} x_{i}^{*}-b_{i}\right)^{\prime} y_{i 1}^{*}=0
$$

and

$$
\left(c_{i}-A_{i i}^{\prime} y_{i 1}^{*}-A_{i+1, i}^{\prime} y_{i+1,1}^{*}+y_{i 2}^{*}\right)^{\prime} x_{i}^{*}=0 .
$$

The next theorem provides a transversality condition that under weak duality guarantees optimality for any pair of primal and dual feasible solutions that satisfy complementary slackness.

Theorem 4.2. Suppose $\tilde{x} \in X, \tilde{y} \in Y$ satisfy complementary slackness. Furthermore, suppose that

$$
\liminf _{i \rightarrow \infty} \tilde{y}_{i+1,1}^{\prime} A_{i+1, i} \tilde{x}_{i}=0 .
$$

Then

$$
C(\tilde{x})=\sum_{i=1}^{\infty} c_{i}^{\prime} \tilde{x}_{i}=\limsup _{N \rightarrow \infty} \sum_{i=1}^{N}\left(\tilde{b}_{i}^{\prime} \tilde{y}_{i 1}-u_{i}^{\prime} \tilde{y}_{i 2}\right)=B(\tilde{y}) .
$$


If, moreover, weak duality holds, then $\tilde{x}$ is an optimal solution for $(\mathrm{P})$, and $\tilde{y}$ is optimal for (D). Futhermore, if

$$
\lim _{i \rightarrow \infty} \tilde{y}_{i+1,1}^{\prime} A_{i+1, i} \tilde{x}_{i}=0
$$

then

$$
B(\tilde{y})=\sum_{i=1}^{\infty}\left(b_{i}^{\prime} \tilde{y}_{i 1}-u_{i}^{\prime} \tilde{y}_{i 2}\right)
$$

Proof. By complementary slackness we have, for all $i$,

$$
\left(c_{i}-A_{i i}^{\prime} \tilde{y}_{i 1}-A_{i+1, i}^{\prime} \tilde{y}_{i+1, i}+\tilde{y}_{i 2}\right)^{\prime} \tilde{x}_{i}=0,
$$

or equivalently

$$
c_{i}^{\prime} \tilde{x}_{i}=\tilde{x}_{i}^{\prime} A_{i i}^{\prime} \tilde{y}_{i 1}+\tilde{x}_{i}^{\prime} A_{i+1, i}^{\prime} \tilde{y}_{i+1,1}-\tilde{x}_{i}^{\prime} \tilde{y}_{i 2} .
$$

Summation over all $i$ on both sides yields

$$
C(\tilde{x})=\sum_{i=1}^{\infty} c_{i}^{\prime} \tilde{x}_{i}=\sum_{i=1}^{\infty}\left(\tilde{x}_{i}^{\prime} A_{i i}^{\prime} \tilde{y}_{i 1}+\tilde{x}_{i}^{\prime} A_{i+1, i}^{\prime} \tilde{y}_{i+1,1}-\tilde{x}_{i}^{\prime} \tilde{y}_{i 2}\right) .
$$

Also by complementary slackness,

$$
\tilde{x}_{i-1}^{\prime} A_{i, i-1}^{\prime} \tilde{y}_{i 1}+\tilde{x}_{i}^{\prime} A_{i i}^{\prime} \tilde{y}_{i 1}=b_{i}^{\prime} \tilde{y}_{i 1}
$$

and

$$
\tilde{x}_{i}^{\prime} \tilde{y}_{i 2}=u_{i}^{\prime} \tilde{y}_{i 2},
$$

so

$$
\begin{aligned}
B(\tilde{y}) & =\limsup _{N \rightarrow \infty} \sum_{i=1}^{N}\left(b_{i}^{\prime} \tilde{y}_{i 1}-u_{i}^{\prime} \tilde{y}_{i 2}\right) \\
& =\limsup _{N \rightarrow \infty} \sum_{i=1}^{N}\left(\tilde{x}_{i-1}^{\prime} A_{i, i-1}^{\prime} \tilde{y}_{i 1}+\tilde{x}_{i}^{\prime} A_{i i}^{\prime} \tilde{y}_{i 1}-\tilde{x}_{i}^{\prime} \tilde{y}_{i 2}\right) \\
& =\limsup _{N \rightarrow \infty}\left(\sum_{i=1}^{N}\left(\tilde{x}_{i}^{\prime} A_{i i}^{\prime} \tilde{y}_{i 1}+\tilde{x}_{i}^{\prime} A_{i+1, i}^{\prime} \tilde{y}_{i+1,1}-\tilde{x}_{i}^{\prime} \tilde{y}_{i 2}\right)-\tilde{x}_{N}^{\prime} A_{N+1, N}^{\prime} \tilde{y}_{N+1,1}\right) \\
& =\sum_{i=1}^{\infty}\left(\tilde{x}_{i}^{\prime} A_{i i}^{\prime} \tilde{y}_{i 1}+\tilde{x}_{i}^{\prime} A_{i+1, i}^{\prime} \tilde{y}_{i+1,1}-\tilde{x}_{i}^{\prime} \tilde{y}_{i 2}\right)-\liminf _{N \rightarrow \infty} \tilde{x}_{N}^{\prime} A_{N+1, N}^{\prime} \tilde{y}_{N+1,1} \\
& =\sum_{i=1}^{\infty}\left(\tilde{x}_{i}^{\prime} A_{i i}^{\prime} \tilde{y}_{i 1}+\tilde{x}_{i}^{\prime} A_{i+1, i}^{\prime} \tilde{y}_{i+1,1}-\tilde{x}_{i}^{\prime} \tilde{y}_{i 2}\right)=C(\tilde{x}) .
\end{aligned}
$$

Since weak duality holds, we have the optimality of $\tilde{x}$ and $\tilde{y}$. Furthermore, if $\lim _{i \rightarrow \infty} \tilde{y}_{i+1,1}^{\prime} A_{i+1, i} \tilde{x}_{i}=0$ 
we can conclude

$$
\begin{aligned}
B(\tilde{y}) & =\limsup _{N \rightarrow \infty} \sum_{i=1}^{N}\left(b_{i}^{\prime} \tilde{y}_{i 1}-u_{i}^{\prime} \tilde{y}_{i 2}\right)=\liminf _{N \rightarrow \infty} \sum_{i=1}^{N}\left(b_{i}^{\prime} \tilde{y}_{i 1}-u_{i}^{\prime} \tilde{y}_{i 2}\right) \\
& =\sum_{i=1}^{\infty}\left(b_{i}^{\prime} \tilde{y}_{i 1}-u_{i}^{\prime} \tilde{y}_{i 2}\right) .
\end{aligned}
$$

Note that the objective function values given for the dual formulation of Sections 1 and 2 are in agreement for $\tilde{y}$ under the conditions of Theorem 4.2.

The following theorem is the main result of the paper.

Theorem 4.3. Suppose the following conditions on (P) hold:

(i) The constraint data $A_{i+1, i}, u_{i}, m_{i}$ and $n_{i}$ for (P) are uniformly bounded over i, i.e., $\left(u_{i}\right)_{j} \leqslant \bar{u}<\infty,\left(A_{i+1, i}\right)_{k l} \leqslant \bar{a}<\infty, m_{i} \leqslant \bar{m}<\infty$ and $n_{i} \leqslant \bar{n}<\infty$ for all $i, j, k, l$,

(ii) Weak duality holds for (P) and (D),

(iii) Transversality holds for (D), i.e.,

$$
\lim _{i \rightarrow \infty} y_{i 1}^{*}=0
$$

for some $y^{*} \in Y^{*}(\infty)$.

Then $y^{*} \in Y^{*}$ and strong duality holds, i.e., there exist optimal solutions $x^{*} \in X^{*}$ and $y^{*} \in Y^{*}$ such that

$$
C\left(x^{*}\right)=B\left(y^{*}\right) \text {. }
$$

Proof. Since $y^{*} \in Y^{*}(\infty)$, there exists a subsequence $\left\{N_{k}\right\}$ such that

$$
\lim _{k \rightarrow \infty} y_{i}^{*}\left(N_{k}\right)=y_{i}^{*}
$$

for all $i$, where $y_{i}^{*}\left(N_{k}\right) \in Y^{*}\left(N_{k}\right)$ for all $k$. Choose $x^{*}\left(N_{k}\right) \in X^{*}\left(N_{k}\right) \cap X$ for all $k$. Then, passing to a subsequence of $\left\{N_{k}\right\}$ if necessary, we have

$$
\lim _{k \rightarrow \infty} x_{i}^{*}\left(N_{k}\right)=x_{i}^{*}
$$

for all $i$ for some $x^{*} \in X$ by the compactness of $\prod_{i=1}^{\infty}\left[0, u_{i}\right]$ in the product topology. By Lemma $4.1, x^{*}$ and $y^{*}$ satisfy complementary slackness. By assumption,

$$
\lim _{i \rightarrow \infty} e^{\prime} y_{i 1}^{*}=0 \text {. }
$$

So we have

$$
\begin{aligned}
y_{i+1,1}^{* \prime} A_{i+1, i} x_{i}^{*} & =\sum_{k=1}^{m_{i+1}} \sum_{l=1}^{n_{i}}\left(y_{i+1,1}^{*}\right)_{k}\left(x_{i}^{*}\right)_{l}\left(A_{i+1, i}\right)_{k l} \\
& \leqslant \sum_{k=1}^{m_{i+1}} \sum_{l=1}^{n_{i}}\left(y_{i+1,1}^{*}\right)_{k} \bar{u} \bar{a} \\
& =n_{i} \bar{u} \bar{a} \sum_{k=1}^{m_{i+1}}\left(y_{i+1,1}^{*}\right)_{k} \\
& \leqslant \bar{n} \bar{u} \bar{a}\left(e^{\prime} y_{i+1,1}^{*}\right),
\end{aligned}
$$


which gives

$$
\liminf _{i \rightarrow \infty} y_{i+1,1}^{* \prime} A_{i+1, i} x_{i}^{*} \leqslant \bar{n} \bar{u} \bar{a} \lim _{i \rightarrow \infty} e^{\prime} y_{i+1,1}^{*}=0 .
$$

So, Theorem 4.2 provides that $x^{*}$ is optimal for (P) and $y^{*}$ is optimal for (D), and the corresponding objective function values are equal.

Remarks. (a) Condition (ii) by Theorem 3.1 may be replaced by the requirement in (i) that $0 \leqslant\left(A_{i+1, i}\right)_{k l} \leqslant \bar{a}<\infty$ for all $i, k, l$. (b) From the proof of Theorem 4.3, we may relax the requirement that $m_{i} \leqslant \bar{m}<\infty$ in (i) by replacing condition (iii) with the requirement that $\lim e^{\prime} y_{i 1}^{*}=0$.

The important condition in Theorem 4.3 for strong duality to hold is the transversality condition that requires (in the language of Schochetman and Smith, 1989) that algorithmically optimal prices of resources available in the $i$ th period go to zero as $i$ goes to infinity. To this point, it is not clear whether any nontrivial instance of $(\mathrm{P})$ satisfies this condition. In the next section, we prove that a classic production planning problem satisfies the transversality condition and hence is an important problem for which duality holds.

\section{An application to production planning over an infinite horizon}

Consider the problem of scheduling production to meet nonstationary demand over an infinite horizon. The problem may be formulated by the following linear program (Denardo, 1982, p. 87):

$$
\min \sum_{i=1}^{\infty}\left(k_{i} P_{i}+h_{i} I_{i}\right) \alpha^{i-1}
$$

subject to

$$
\begin{aligned}
& I_{i-1}+P_{i}-I_{i} \geqslant d_{i} \quad(i=1,2, \ldots), \\
& 0 \leqslant P_{i} \leqslant \bar{P}_{i} \quad(i=1,2, \ldots), \\
& 0 \leqslant I_{i} \leqslant \bar{I}_{i} \quad(i=1,2, \ldots),
\end{aligned}
$$

where $I_{j}$ is the net inventory ending period $j$ with $I_{0}=0, P_{j}$ is the production in period $j, D_{j}$ is the demand for production in period $j, k_{j}$ is the production cost and $h_{j}$ is the inventory holding cost for period $j, j=1,2, \ldots$ The factor $\alpha$ is the discount factor reflecting the time value of money where $0<\alpha<1$. The dual (D) becomes

$$
\sup \limsup _{N \rightarrow \infty} \sum_{i=1}^{N}\left(d_{i} w_{i}-\bar{P}_{i} u_{i}-\bar{I}_{i} v_{i}\right)
$$


subject to

$$
\begin{aligned}
& w_{i}-u_{i} \leqslant k_{i} \alpha^{i-1} \quad(i=1,2, \ldots), \\
& -w_{i}+w_{i+1}-v_{i} \leqslant h_{i} \alpha^{i-1} \quad(i=1,2, \ldots), \\
& w_{i}, u_{i}, v_{i} \geqslant 0 \quad(i=1,2, \ldots) .
\end{aligned}
$$

Note that without loss of optimality, we have that demand is met exactly, i.e., $I_{i-1}+P_{i}-I_{i}=d_{i}$ for all $i$, in program (Q).

As in Schochetman and Smith (1992), we make the following assumptions:

\section{Assumptions.}

(i) $\quad \inf _{i}\left(\bar{P}_{i}-d_{i}\right)>0$.

(ii) $\tilde{P}_{i} \leqslant \bar{P}<\infty$ for all $i$,

$$
\bar{I}_{i} \leqslant \bar{I}<\infty \text { for all } i \text {. }
$$

(iii) $k_{i}, h_{i} \geqslant 0$ and $\max \left(k_{i}, h_{i}\right) \leqslant G \gamma^{i}$ for all $i$ for some $0<G<\infty, 0<\gamma<1 / \alpha$.

Note that (Q) is of the form (P) under the identification $x_{i}=\left(P_{i} I_{i}\right)^{\prime}, c_{i}=\left(k_{i} h_{i}\right) \alpha^{i-1}$, $A_{i, i-1}=\left(\begin{array}{ll}1 & 0\end{array}\right), A_{i i}=(1-1), b_{i}=d_{i}$, and $\mu_{i}=\left(\bar{P}_{i} \bar{I}_{i}\right)^{\prime}$.

It is easily verified that Assumptions $\mathrm{A}$ and $\mathrm{B}$ of Section 1 are satisfied. Moreover, since the off diagonal submatrices $A_{i-1, i}=\left(\begin{array}{ll}1 & 0\end{array}\right) \geqslant 0$ for all $i$, we have by Theorem 3.1 that under assumptions (i), (ii) and (iii), weak duality holds for (Q) and therefore there is no duality gap by Theorem 3.2. Verification of the transversality conditions for strong duality will be considerably more difficult. We begin by establishing that we may restrict consideration without loss of optimality to a bounded subset of the feasible solutions to (D) and $(\mathrm{D}(N))$.

Set $y_{i}=\left(w_{i}, u_{i}, v_{i}\right) \in \mathbb{R}^{3}$ and $y=(w, u, v)$.

Lemma 5.1. For the Production Planning Problem (Q), there exists $\bar{y}_{i}<\infty$ for all $i$, such that

$$
\sup _{y \in \bar{Y}} B(w, u, v)=\sup _{y \in Y} B(w, u, v) .
$$

where $\bar{Y}=\left\{y \in Y: y_{i} \leqslant \bar{y}_{i}\right.$ for all $\left.i\right\}$. Moreover, for all $N$,

$$
\sup _{y \in \bar{Y}(N)} B(w, u, v ; N)=\sup _{y \in Y(N)} B(w, u, v ; N),
$$

where $\bar{Y}(N)=\left\{y \in Y(N): y_{i} \leqslant \bar{y}_{i}\right.$ for all $\left.i\right\}$.

Proof. See Appendix.

Lemma 5.2. For the Production Planning Problem (Q), suppose $y(N) \in \bar{Y}(N)$ for all N. Suppose further that

$$
\lim _{k \rightarrow \infty} w_{i}\left(N_{k}\right)=w_{i}
$$


exists for all $i$ for some subsequence $\left\{N_{k}\right\}$ of the positive integers. If

$$
\limsup _{i \rightarrow \infty} w_{i}<0 \text {, }
$$

then

$$
\liminf _{N \rightarrow \infty} B(y(N) ; N)=-\infty .
$$

Proof. See Appendix.

We are now ready to prove the main result of this section.

Theorem 5.3. Suppose the production Planning Problem (Q) satisfies Assumptions (i), (ii), and (iii). Then weak and strong duality hold for (Q) and (D), i.e.,

$$
B(y) \leqslant C(x)
$$

for all $x \in X$ and $y \in Y$ and there exist $x^{*} \in X^{*}$ and $y^{*} \in Y^{*}$ such that

$$
B\left(y^{*}\right)=C\left(x^{*}\right) \text {. }
$$

Proof. Consider any sequence $\left\{y^{*}(N)\right\}, y^{*}(N) \in Y^{*}(N)$ for all $N$. Without loss of generality we can assume $y^{*}(N) \in \bar{Y}$ for all $N$. Since $\bar{Y}$ lies in the product of compact sets, it is compact in the product topology. Hence there exists a subsequence of $\left\{y^{*}(N)\right\}$ converging to some $y^{*} \in \bar{Y}$. Since no duality gap exists for $(\mathrm{Q})$, we have by Theorem 3.2 that $\lim _{N \rightarrow \infty} B\left(y^{*}(N) ; N\right)=B^{*}=C^{*}>-\infty$. Thus, by Lemma 5.2,

$$
\lim _{i \rightarrow \infty} w_{i}^{*}=0 .
$$

Hence conditions (i) through (iii) of Theorem 4.3 are satisfied (with $\bar{u}=\max (\bar{P}, \bar{I})$, $\bar{a}=1$ and $\bar{n}=2$ ). We conclude that strong duality holds for the production planning problem.

Hence weak and strong duality hold for the Production Planning Problem under very mild regularity conditions. One might expect the same results under comparable conditions for a wide variety of investment planning problems including equipment replacement and capacity expansion.

\section{Conclusions}

We have established weak and strong duality for a large class of doubly infinite linear programs under the key transversality condition that dual prices asymptotically converge to zero. Moreover this transversality condition was shown to be met by a nonstationary infinite horizon production planning problem.

Using weak duality, one can bound the optimal primal value thus providing a measure of error to approximate solutions to $(\mathrm{P})$. Moreover, under strong duality, it becomes possible in principle to analytically establish optimality of a candidate feasible primal solution by demonstrating equality in value with a candidate feasible dual solution. 


\section{Appendix}

Lemma 5.1. For the Production Planning Problem (Q), there exist $\bar{y}_{i}<\infty$ for all $i$, such that

$$
\sup _{y \in \bar{Y}} B(w, u, v)=\sup _{y \in Y} B(w, u, v)
$$

where $\bar{Y}=\left\{y \in Y: y_{i} \leqslant \bar{y}_{i}\right.$ for all $\left.i\right\}$. Moreover, for all $N$,

$$
\sup _{y \in \bar{Y}(N)} B(w, u, v ; N)=\sup _{y \in Y(N)} B(w, u, v ; N)
$$

where $\bar{Y}(N)=\left\{y \in Y(N): y_{i} \leqslant \bar{y}_{i}\right.$ for all $\left.i\right\}$.

Proof. We will prove that, for all $i$, there exist $\bar{w}_{i}<\infty$ such that for all $N \geqslant i$ the following holds:

$$
\sup _{y \in Y(N) ; w_{i} \geqslant \bar{w}_{i}} B(w, u, v ; N)<0 .
$$

Combining this with

$$
\sup _{y \in Y(N)} B(w, u, v ; N)=B^{*}(N) \geqslant 0,
$$

we can conclude that

$$
\sup _{y \in Y(N)} B(w, u, v ; N)=\sup _{y \in Y(N) ; w_{i} \leqslant \bar{w}_{i}} B(w, u, v ; N)
$$

for all $i$ and $N \geqslant i$. Furthermore, we will have that

$$
\sup _{y \in Y} B(w, u, v)=\sup _{y \in Y ; w_{i} \leqslant \bar{w}_{i}} B(w, u, v)
$$

for all $i$.

Choose some index $i$. Then, for all $N \geqslant i$,

$$
\begin{aligned}
B(w, u, v ; N) & =\sum_{j=1}^{N}\left(d_{j} w_{j}-\bar{P}_{j} u_{j}-\bar{I}_{j} v_{j}\right) \\
& \leqslant \sum_{j=1}^{N}\left(d_{j} w_{j}-\bar{P}_{j} w_{j}+\bar{P}_{j} w_{j}-\bar{P}_{j} u_{j}\right) \\
& =\sum_{j=1}^{N}\left(\left(d_{j}-\bar{P}_{j}\right) w_{j}+\bar{P}_{j}\left(w_{j}-u_{j}\right)\right) \\
& \leqslant\left(d_{i}-\bar{P}_{i}\right) w_{i}+\sum_{j=1}^{N} \bar{P}_{j} k_{j} \alpha^{j-1} \\
& \leqslant\left(d_{i}-\bar{P}_{i}\right) w_{i}+\sum_{j=1}^{N} \bar{P}_{G} \gamma(\alpha \gamma)^{j-1} \\
& \leqslant\left(d_{i}-\bar{P}_{i}\right) w_{i}+\frac{\bar{P} G \gamma}{1-\alpha \gamma} .
\end{aligned}
$$


Thus, choosing e.g.,

$$
\bar{w}_{i}=\frac{\bar{P} G \gamma}{(1-\alpha \gamma)\left(\bar{P}_{i}-d_{i}\right)}+1<\infty,
$$

we get

$$
\sup _{y \in Y(N) ; w_{i} \geqslant \bar{w}_{i}} B(w, u, v ; N) \leqslant\left(d_{i}-\bar{P}_{i}\right) \bar{w}_{i}+\frac{\bar{P} G \gamma}{1-\alpha \gamma}=d_{i}-\bar{P}_{i}<0
$$

for $i \leqslant N$. Since inequality (1) holds for all $N \geqslant i$, we also have

$$
B(w, u, v)=\limsup _{N \rightarrow \infty} B(w, u, v ; N) \leqslant\left(d_{i}-\bar{P}_{i}\right) w_{i}+\frac{\bar{P} G \gamma}{1-\alpha \gamma}
$$

and thus

$$
\sup _{y \in Y ; w_{i} \geqslant \tilde{w}_{i}} B(w, u, v)<0 .
$$

We can now conclude that for all $N$,

$$
\sup _{y \in Y(N) ; w_{i} \leqslant \bar{w}_{i}} B(w, u, v ; N)=\sup _{y \in Y(N)} B(w, u, v ; N)
$$

and

$$
\sup _{y \in Y ; w_{i} \leqslant \bar{w}_{i}} B(w, u, v)=\sup _{y \in Y} B(w, u, v) .
$$

Moreover, combining the results for all indices $i$,

$$
\sup _{y \in Y(N) ; w_{i} \leqslant \bar{w}_{i} \forall i} B(w, u, v ; N)=\sup _{y \in Y(N)} B(w, u, v ; N)
$$

and

$$
\sup _{y \in Y ; w_{i} \leqslant \vec{w}_{i} \forall i} B(w, u, v)=\sup _{y \in Y} B(w, u, v) .
$$

Now, observe that in an optimal solution we will have

$$
\begin{aligned}
& u_{i}=\max \left(0, w_{i}-k_{i} \alpha^{i-1}\right), \\
& v_{i}=\max \left(0,-w_{i}+w_{i+1}-h_{i} \alpha^{i-1}\right),
\end{aligned}
$$

so

$$
\begin{aligned}
& u_{i} \leqslant \max \left(0, \bar{w}_{i}-k_{i} \alpha^{i-1}\right)=: \bar{u}_{i}, \\
& v_{i} \leqslant \max \left(0, \bar{w}_{i+1}-h_{i} \alpha^{i-1}\right)=: \bar{v}_{i} .
\end{aligned}
$$

Combining the results gives

$$
\sup _{y \in Y(N)} B(y ; N)=\sup _{y \in \bar{Y}(N)} B(y ; N)
$$

for all $N$, and

$$
\sup _{y \in Y} B(y)=\sup _{y \in \bar{Y}} B(y) .
$$


Lemma 5.2. For the Production Planning Problem (Q), suppose $y(N) \in \bar{Y}(N)$ for all N. Suppose further that

$$
\lim _{k \rightarrow \infty} w_{i}\left(N_{k}\right)=w_{i}
$$

exist for all $i$ for some subsequence $\left\{N_{k}\right\}$ of the positive integers. If

$$
\lim \sup w_{i}>0
$$

then

$$
\liminf _{N \rightarrow \infty} B(y(N) ; N)=-\infty
$$

Proof. By hypothesis, there is a subsequence $\left\{i_{j}\right\}$ such that

$$
\lim _{j \rightarrow \infty} \lim _{k \rightarrow \infty} w_{i_{j}}\left(N_{k}\right) \geqslant \delta>0 .
$$

Assume without loss of generality that $\delta<\infty$. This means:

$$
\forall 0<\varepsilon<\delta, \exists J_{\varepsilon} \text { such that for all } j \geqslant J_{\varepsilon}: \quad \lim _{k \rightarrow \infty} w_{i_{j}}\left(N_{k}\right) \geqslant \delta-\varepsilon>0
$$

and

$$
\begin{aligned}
& \forall 0<\varepsilon<\delta, \exists J_{\varepsilon} \text { such that for all } j \geqslant J_{\varepsilon}: \\
& \forall 0<\eta<\delta-\varepsilon, \exists K_{\eta}(j) \text { such that for all } k \geqslant K_{\eta}(j): \\
& \quad w_{i_{j}}\left(N_{k}\right) \geqslant \delta-\varepsilon+\eta>0 .
\end{aligned}
$$

Choose some fixed $0<\varepsilon<\delta$, and $0<\eta<\delta-\varepsilon$. Let $\mu=\delta-\varepsilon-\eta$. Without loss of generality we assume $K_{\eta}(j+1)>K_{\eta}(j)$ for all $j$. Now define

$$
\mathscr{J}_{k}:=\left\{j \geqslant J_{\varepsilon} \mid w_{i_{j}}\left(N_{k}\right) \geqslant \mu, i_{j} \leqslant N_{k}\right\} \text {. }
$$

Choose a positive integer $M$, arbitrary. Then, for $k \geqslant K_{\eta}\left(J_{\varepsilon}+M-1\right)$ we have $\left|\mathscr{J}_{k}\right| \geqslant M$. Thus, since $M$ was chosen arbitrarily, we can conclude

$$
\lim _{k \rightarrow \infty}\left|\mathscr{I}_{k}\right|=\infty \text {. }
$$

Now we have, for all $k$,

$$
\begin{aligned}
B\left(y\left(N_{k}\right) ; N_{k}\right) & =\sum_{i=1}^{N_{k}}\left(d_{i} w_{i}\left(N_{k}\right)-\bar{P}_{i} u_{i}\left(N_{k}\right)-\bar{I}_{i} v_{i}\left(N_{k}\right)\right) \\
& \leqslant \sum_{i=1}^{N_{k}}\left(\left(d_{i}-\bar{P}_{i}\right) w_{i}\left(N_{k}\right)+\bar{P}_{i}\left(w_{i}\left(N_{k}\right)-u_{i}\left(N_{k}\right)\right)\right) \\
& \leqslant \sum_{i=1}^{N_{k}}\left(d_{i}-\bar{P}_{i}\right) w_{i}\left(N_{k}\right)+\sum_{i=1}^{N_{k}} \bar{P}_{i} k_{i} \alpha^{i-1} \\
& \leqslant-\sigma \sum_{i=1}^{N_{k}} w_{i}\left(N_{k}\right)+\frac{\bar{P} G \gamma}{1-\alpha \gamma} \\
& \leqslant-\sigma \mu\left|\mathscr{I}_{k}\right|+\frac{\bar{P} G \gamma}{1-\alpha \gamma} .
\end{aligned}
$$


Thus:

$$
\begin{aligned}
\liminf _{N \rightarrow \infty} B(y(N) ; N) & \leqslant \lim _{k \rightarrow \infty} B\left(y\left(N_{k}\right) ; N_{k}\right) \\
& \leqslant \lim _{k \rightarrow \infty}\left(-\sigma \mu\left|\mathscr{J}_{k}\right|+\frac{\bar{P} G \gamma}{1-\alpha \gamma}\right) \\
& =\frac{\bar{P} G \gamma}{1-\alpha \gamma}-\sigma \mu \lim _{k \rightarrow \infty}\left|\mathscr{J}_{k}\right|=-\infty .
\end{aligned}
$$

\section{References}

E.J. Anderson and P. Nash, Linear Programming in Infinite-dimensional Spaces (Wiley, New York, 1987).

J.C. Bean and R.L. Smith, "Conditions for the existence of planning horizons," Mathematics of Operations Research 9 (1984) 391-401.

A. Ben-Israel, A. Charnes and K. Kortanek, "Duality and asymptotic solvability over cones," Bulletin of the American Mathematical Society 75 (1969) 318-324.

C. Bès and S. Sethi, "Concepts of forecast and decision horizons: Applications to dynamic stochastic optimization problems," Mathematics of Operations Research 13 (1988) 295-310.

J.M. Borwein, "Direct theorems in semi-infinite convex programming," Mathematical Programming 21 (1981) 301-318.

J.M. Borwein, "Semi-infinite programming duality: How special is it?," in: A.V. Fiacco and K.O. Kortanek, eds, Semi-Infinite Programming and Applications (Springer, Berlin, 1983).

A. Charnes, W.W. Cooper and K.O. Kortanek, "Duality in semi-infinite programs and some works of Haar and Caratheodory," Management Science 9 (1963) 209-228.

A. Charnes, P.R. Gribek and K.O. Kortanek, "Separably Infinite Programs," Zeitschrift für Operations Research 24 (1980) 33-45.

E.V. Denardo, Dynamic Programing: Models and Applications (Prentice-Hall, Englewood Cliffs, NJ, 1982).

R.J. Duffin, "Infinite programs," in: H.W. Kuhn and A.W. Tucker, eds, Linear Inequalities and Related Systems (Princeton Univeristy Press, Princeton, NJ, 1956).

R.J. Duffin, R.G. Jeroslow and L.A. Karlovitz, "Duality in semi-infinite linear programming," in: A.V. Fiacco and K.O. Kortanek, eds, Semi-Infinite Programming and Applications (Springer, Berlin, 1983).

R.J. Duffin and L.A. Karlovitz, "An infinite linear program with a duality gap," Management Science 12 (1965) 122-134.

J. Evers, Linear Programming over an Infinite Horizon (Tilburg University Press, Tilburg, Netherlands, 1973).

J. Evers, "A duality theory for infinite horizon optimization of concave input/output processes," Mathematics of Operations Research 8 (1983) 479-497.

R. Grinold, "Infinite horizon programs," Management Science 18 (1971) 157-170.

R. Grinold, "Finite horizon approximations of infinite horizon linear programs," Mathematical Programming 12 (1977) 1-17.

R. Grinold, "Convex infinite horizon programs," Mathematical Programming 25 (1983) 64-82.

R. Grinold and D.S.P. Hopkins, "Computing optimal solutions for infinite horizon mathematical programs with a transient stage," Operations Research 21 (1972) 179-187.

D.S.P. Hopkins, "Infinite horizon optimality in an equipment replacement and capacity expansion model," Management Science 18 (1971) 145-156.

P. Jones, J. Zydiak and W. Hopp, "Stationary dual prices and depreciation," Mathematical Programming 41 (1988) 357-366.

J.F. Karney, "Duality gaps in semi-infinite linear programming: An approximation problem," Mathematical Programming 20 (1981) 129-143.

D.G. Luenberger, Optimization by Vector Space Methods (Wiley, New York, 1969).

J. Ponstein, Approaches to the Theory of Optimization (Cambridge University Press, Cambridge, 1980).

I.E. Schochetman and R.L. Smith, "Infinite horizon optimization", Mathematics of Operations Research 14 (1989) 559-574.

I.E. Schochetman and R.L. Smith, "Finite dimensional approximation in infinite dimensional programming," to appear in: Mathematical Programming (1992). 